\title{
ASYMPTOTIC STABILITY OF BAYESIAN UPDATING FOR SPENCIAN EXAMPLES *
}

\author{
Peter H. HUANG ** \\ University of Iowa, Ames, IA 50011, USA \\ Harvard University, Cambridge, MA 02138, USA \\ Stanford University, Stanford, CA 94305, USA \\ Received 28 June 1984
}

\begin{abstract}
Under the assumption of a common prior over a signalling cost parameter, employers revising their beliefs in a Bayesian fashion are shown to converge to a separating equilibrium of Spence's (1974b) continuous ability model. Following him, specific functional forms are used.
\end{abstract}

\section{Introduction}

In a volatile dynamic market (for example a growing, as opposed to a mature, industry), noisy exogenous disturbances perturb the signalling costs held fixed in Spence's (1974b) model. Shifting of employees' tastes randomly influences their utilities. For instance, children in one generation might watch more television, play video games, or alternatively return to being more academically oriented. In any event, whatever the nature of the shock, in order to capture phenomena of the above category a cohort variable is added to the basic Spencian (1974a) formulation. The natural economic stories given above suggest it should not be varying across the population of a given time period (at any rate significantly less than it does across generations). So, this is not the kind of analysis found in chapter 6 of Spence's (1974a) thesis, in which parents' incomes are differing in their impact both over time and across individuals of the same age. Examples of educational costs correlated imperfectly with ability in a negative way are interesting, but difficult. His only closed-form solution of such a case is one involving constant marginal costs which are jointly normally distributed with ability. Non-negativity problems aside, the noisy costs model is quite descriptive of the stylized facts. In this paper though, a different approach involving exogenously generated noise is adopted.

\section{A continuous ability example with uniformly wrong priors}

Employers will be assumed to have common knowledge of their potential employees' optimization problems. Thus, employers realize that individuals choose education to maximize their net income,

* This is a shortened version of chapter two from the author's thesis, Asymptotic and structural stability of signalling equilibria, for a Ph.D. in applied mathematics at Harvard University. The author is currently a visitor at the University of lowa.

** I thank my dissertation committee members: K.J. Arrow, J.R. Green, and Y.C. Ho; as well as J.L. Solow, T. Ichiishi, and D. Malueg from the University of Iowa Economics Department for their useful discussions: in addition, thanks to Mia, Pearl, and Milt. 
where net income is just wages indexed by schooling minus the cost of acquiring that schooling. As companies observe market wages, in order that residual uncertainty remains about which ability types choose which signals, it must be that signalling cost functions are privately known. In a Bayesian spirit it will be that all these cost functions belong to some family with a parameter that has to be estimated. So as to avoid chasing a moving target, I have set up the learning problem so that the real driving source of uncertainty is exogenously generated. This aggregate parameter $\theta$ is randomly distributcd over timc with finite mean $\theta^{*}$ unknown to managers. Define: $n$ is an unobservable fixed attribute such as natural ability, $y$ is some observable individually chosen signal, and $s$ is individual marginal productivity, a function of $n$ and $y$, so that

$s=S(n, y)$ where $n \in(0, N]$ and $y \in[0, Y]$

$C(n, y)=$ cost of buying signal $y$ for type $n$ individuals.

Furthermore, in order to insure that a static informational equilibrium exists, certain restrictions are improved on these functions, namely, $S_{n}>0, S_{y} \geqslant 0, C_{y}>0$, and $C_{y n}<0$. These monotonicity conditions were shown by Spence ${ }^{1}$ to guarantee a self-realizing belief about $s$ given $y$. Let there be a parameter $\theta$ in signal costs: $C(n, y, \theta)$, where $\theta$ has a known distribution with unknown mean $\theta^{*}$. Besides ignorance about $\theta^{*}$, I assume $C(n, y, \theta)$ and $S(n, y)$ are known to firms in so much as once the correct value of $\theta^{*}$ was known, employers themselves could actually solve the individuals' optimization problems. Based on subjective beliefs about $\theta^{*}$, employers form their expectations concerning productivity given a fixed level of education $y$. After offering some resulting trial wage schedule, individuals respond (instantaneously) with their actual $\theta_{t}$. Hence $\theta$ is a random variable having a fixed mean value $\theta^{*}$ and each period $\theta_{t}=\theta^{*}+\epsilon_{t}$, where $\epsilon_{t}$ is a sequence of i.i.d. random variables (r.v.'s) having mean 0 , and some finite variance.

In particular, suppose these are common knowledge amongst firms:

$$
\begin{array}{ll}
S(n, y)=n y^{\alpha}, & \alpha \in[0,1), \\
C\left(n, y, \theta_{t}\right)=\theta_{t} y / n, & \theta_{t}>0 .
\end{array}
$$

Notice that $\alpha \geqslant 0$ to satisfy $S_{y} \geqslant 0$. The reason for $\alpha$ to be less than one is due to the second-order condition of the individuals' problem: maximize $W(y)-C(n, y, \theta)$. Now I can formally state the first proposition about competitive Bayesian employers facing the particular environment described by the aforementioned functional specifications:

Proposition 1. If there is a common prior over $\theta$ shared by all employers, and individual worker productivity is observable ex post then, independent of the particular history of the samples hired, all firms converge with probability one to the true $\theta^{*}$ value.

Proof. The first order condition (f.o.c.) $W_{t}^{\prime}(y)=C_{y}(n, y, \theta)$ in this present special case is: $W_{t}^{\prime}(y)=$ $\theta_{i / n}$, and so

$$
n=\frac{\theta_{t}}{W_{t}^{\prime}(y)},
$$

\footnotetext{
${ }^{1}$ See theorem 1 in Spence (1974a, pp. 303-304) for a proof of this fact if additionally there is a $y^{*}$ such that for all $y>y^{*}$, $C y>S y$.
} 
expressing $n$ implicitly as a function of $\theta$ and $y$. Thus, in order for a $W(y)$ to be a candidate for an equilibrium wage offer it has to be that $W_{t}(y)=\mathrm{E}_{t}(s \mid y)$ or

$$
\begin{aligned}
& W_{t}(y)=\frac{\mathrm{E}_{t}\left(\theta^{*}\right) y^{\alpha}}{W_{t}^{\prime}(y)}, \quad \text { which means, upon integrating, that } \\
& {\left[W_{t}(y)\right]^{2}=M+\frac{2 \mathrm{E}_{t}\left(\theta^{*}\right) y^{\alpha+1}}{\alpha+1},}
\end{aligned}
$$

where $M$ is a constant of indefinite integration to be determined now. As $W_{t}(0)=\mathrm{E}_{t}(s \mid 0)=0$ it follows that $0=W_{t}^{2}(0)=M$, or $M=0$ and so

$$
W_{t}(y)=\left[\frac{2 \mathrm{E}_{t}\left(\theta^{*}\right) y^{\alpha+1}}{\alpha+1}\right]^{1 / 2}
$$

By $\mathrm{E}_{t}\left(\theta^{*}\right)$ I mean a subjective mean expected value for $\theta_{t}$. I think of Bayesian employers with a common ${ }^{2}$ prior on the mean $\theta^{*}$, namely $p_{0}\left(\theta^{*}\right)$. Then $\mathrm{E}_{t}\left(\theta^{*}\right)=\int \theta^{*} p_{t}\left(\theta^{*}\right) \mathrm{d} \theta^{*}$. Given $W_{t}(y)$, individuals then choose $y$ to maximize net incomc, $W_{l}(y)-\theta_{t} y / n$ and if I define

$A_{t}=\left[\frac{2 \mathrm{E}_{t}\left(\theta^{*}\right)}{\alpha+1}\right]^{1 / 2}, \quad$ the f.o.c. becomes

$$
\frac{A_{t}(\alpha+1) y^{(\alpha-1) / 2}}{2}=\frac{\theta_{t}}{n} \text {, }
$$

$n=\frac{2 \theta_{t} y^{(1-\alpha) / 2}}{(\alpha+1) A_{t}}, \quad$ so that

$s=\frac{2 \theta_{t} y^{(1-\alpha) / 2}}{(\alpha+1) A_{t}} y^{\alpha}=\frac{2 \theta_{t}}{(\alpha+1) A_{t}} y^{(\alpha+1) / 2}$,

$s=\frac{2 \theta_{t}(\alpha+1)^{1 / 2}}{(\alpha+1)\left[2 \mathrm{E}_{t}\left(\theta^{*}\right)\right]^{1 / 2}} y^{(\alpha+1) / 2}=\left[\frac{2}{\alpha+1}\right]^{1 / 2} \frac{\theta_{t}}{\left[\mathrm{E}_{t}\left(\theta^{*}\right)\right]^{1 / 2}} y^{(\alpha+1) / 2}$.

Thus, if employers can observe individual marginal productivity ex post, they can invert for the unobserved realized $\theta_{t}$ since they know $\alpha, y$, and $\mathrm{E}_{t}\left(\theta^{*}\right)$. Thus, by using Komolgorov's law of large numbers for this now conventional Bayesian updating set-up of learning, I obtain the convergence result. Q.E.D.

Furthermore, to start with the simplest possible kind of production technology, let me postulate that aggregate total output $\chi$ is a linear, additively separable function of labour differentiated by signals. ${ }^{3}$ Such a hypothesis concerning the relationship between an individual's contribution to, and the collective sum of, company output, facilitates:

2 Different priors have been considered in chapter 2, section 5 of the author's (1984) dissertation.

3 This assumption means that marginal productivity is a constant, regardless of the amount hired. 
Proposition 2. If all the conditions above hold, then merely being able directly to observe a natural statistic like total production is still enough for beliefs on $\theta$ to converge asymptotically almost surely (a.s.) to $\theta^{*}$.

Proof. A firm hiring a sample of individuals with educational credentials $y_{1}, \ldots, y_{m}$, has a gross product of the sum of individually fixed marginal products, weighted by the number employed of each type of signal as expressed here:

$\chi_{t}=\sum_{1}^{m} s_{j t} N_{j t}, \quad$ where

$N_{j t}=$ number of those hired in period $t$ with education $y_{j}$, and

$\chi_{t}=\sum_{1}^{m} N_{j t}\left[\frac{2 \theta_{t}}{(\alpha+1) A_{t}} y_{j}^{(\alpha+1) / 2}\right]=\frac{2 \theta_{t}}{(\alpha+1) A_{t}} \sum_{1}^{m} N_{j r} y_{j}^{(\alpha+1) / 2}$

Now, as the firm knows $\alpha, N_{j t}, y_{j}$, and $A_{t}$, it can once again solve for unknown $\theta_{t}$. Thus although employers have a common prior here, they can, and probably do, hire different samples $y_{1}, \ldots, y_{m}$ of educational choices. Even with this seemingly differential information, they can all construct a common observation of $\theta_{t}$. Thus, once again by the strong law of large numbers, our stated conclusion follows by a routine application. Q.E.D.

\section{Job allocation decisions}

It will now be asked whether similar conclusions hold for Spence's (1974b) example involving a job allocation decision. In order to have analytically closed form solutions for expectational disequilibria or equilibria, I shall resort once again to special forms of the productivity and cost of signalling functions. Now $s$ is dependent on $y, n$ and some job allocation variable $d$ that is chosen by the firm. With judicious malice aforethought, let us consider the following,

$S(n, y, d)=(16 / 9) d(n-d) y^{2 \alpha}$ with $\alpha \in[0,1)$.

Then maximizing $\mathrm{E}(S(n, y, d) \mid y)$ over the set of all possible real $d$, involves a routine f.o.c. of setting $d$ equal to $0.5 \mathrm{E}(n \mid y)$. This optimal choice of $d$ is naturally dependent on the subjective mean assessment of which $n$ choose $a$ given $y$. Ultimately such beliefs will be related to one's prior distribution of $\theta$. Given the above cost of signalling functions again, which is assumed to be known to employes up to current $\theta$ parameter value, firms can mimic individuals' optimization calculations. Such an exercise as performed above by an 'outside analyst observing market signalling' enables employers to figure out $\mathrm{E}(n \mid y)$. Now, analogous to the examples in the previous section, a convergence result can be proven along the methodology of the cases without a job allocation variable $d$. Ergo, I get the following:

Proposition 3. If $S(n, y, d)$ and $C(n, y, \theta)$ are given as here, then Bayesian firms can decide on a subjectively optimal $d$ for each level of $y$ which over time in the limit will approach the objectively best choice. Indeed, no experimental design problems of conflict between short-run profits and long-term learning exists here in this very special case. 
Proof. Employers offer trial wages of the following kind as given below,

$$
W(y)=\frac{4\left[\mathrm{E}(\theta) y^{\alpha}\right]^{2}}{9\left[W^{\prime}(y)^{2}\right]}, \quad(9 / 4) W W^{\prime 2}=\left[\mathrm{E}(\theta) y^{\alpha}\right]^{2}
$$

which, upon taking square roots on both sides and integrating, yields

$$
W^{3 / 2}=K+\frac{\mathrm{E}(\theta) y^{\alpha+1}}{\alpha+1}
$$

where $K$ is the arbitrary constant of integration found to be zero from the initial condition of $S(n, 0, d)=0$ for all $n$ and $d$ choices. But $\mathrm{I}$ have now reduced the problem to an earlier solved one. Q.E.D.

Granted, all of the above results are quite special in their hypotheses. Especially stringent is the informational requirement on managers actually knowing the particular functional forms I used. These results are shown to hold for a somewhat wider class of functions in Huang (1984a). Additionally, the related structural stability of such signalling equilibria is proved there. Also, Kreps (1984) proves the stability in the strategic sense of Kohlberg and Mertens (1982) of the unique Pareto dominant separating equilibrium of a Spencian labor market example with just a pair of ability levels but a continuum of educational choices. Finally, Huang (1984b) explores another notion of stability, that with respect to 'blocking coalitions' of mechanisms designed by an informed principal for the Spence labor market.

\section{References}

Huang, P.H., 1984a, Structural stability of signalling equilibria, Social Sciences working paper no. 8406 (Graduate College, University of Iowa, Ames, IA) April.

Huang, P.H., 1984b, Incentive efficiency of the Pareto undominated signalling equilibrium, presented at the Winter Econometric Society meetings, Dec.

Kohlberg, E. and J.F. Mertens, 1982, On the strategic stability of equilibria, Mimeo., CORE division paper no. 8248, Nov,

Kreps, D.M., 1984, Signalling games and stable equilibria, preliminary version (Stanford University, Stanford, CA). Presented at The Bayesian Analysis in Economics and Game Theory Workshop (The Institute for Mathematics and Its Applications, University of Minnesota, Minneapolis, MN) Feb.

Spence, A.M., 1974a, Market signalling: Informational transfer in hiring and related processes (Harvard University Press, Cambridge, MA).

Spence, A.M., 1974b, Competitive and optimal responses to signals: An analysis of efficiency and distribution, Journal of Economic theory $7,296-332$. 